\title{
Vitamin-D Deficiency and Myelofibrosis: A Rare but Reversible Association
}

\author{
Akanksha C. Parikh ${ }^{1}$ Tanu Singhal ${ }^{1}$ Zalak Upadhyay ${ }^{1}$ \\ ${ }^{1}$ Department of Pediatrics, Kokilaben Dhirubhai Ambani Hospital, \\ Mumbai, Maharashtra, India \\ J Child Sci 2021;11:e18-e19. \\ Address for correspondence Akanksha C. Parikh, MD, DNB, \\ Paediatrics, Fellowship Paediatric and Adolescent Endocrinology \\ (RGUHS), Kokilaben Dhirubhai Ambani Hospital and Medical Research \\ Institute, Mumbai, 400053, India \\ (e-mail: gandhi.akanksha@gmail.com).
}

Coexistence of vitamin-D deficiency rickets and iron deficiency anemia has been described in the past. ${ }^{1}$ Occasionally, a more serious hematological condition, such as myelofibrosis, can occur in association with vitamin-D deficiency. We present a toddler with rickets due to vitamin-D deficiency and associated myelofibrosis which responded to oral cholecalciferol therapy.

A 16-month-old girl presented with failure to thrive and progressive lower limb deformities noticed since she was 6 months old. She was born of a nonconsanguineous union at term, had a birth weight of $2.5 \mathrm{~kg}$ and was appropriately weaned. She had delayed motor development and on enquiry, there was no past history of seizures, diarrhea, polyuria, jaundice, or blood transfusions. She was a playful child who weighed $7 \mathrm{~kg}$ ( -2.93 standard deviation [SD]) with a length of $67 \mathrm{~cm}$ ( -4.25 SD). Pallor, umbilical hernia, and features of rickets along with anterolateral bowing of bilateral lower limbs and generalized hypotonia were observed. Additionally, a soft hepatomegaly of $2 \mathrm{~cm}$ (span, $10 \mathrm{~cm}$ ) and a firm splenomegaly of $7 \mathrm{~cm}$ along the long axis was found; rest of the systemic examination was unremarkable. The possible differential diagnoses considered were rickets in an infant with a storage disorder or less likely a hematological neoplasm.

Blood and radiologic investigations confirmed vitamin-D deficiency rickets (serum calcium: $8.4 \mathrm{mg} / \mathrm{dL}$, serum phosphorus: $1.9 \mathrm{mg} / \mathrm{dL}$, alkaline phosphatase: $1,136 \mathrm{IU} / \mathrm{L}, 25$-hydroxy vitamin D: $11 \mathrm{ng} / \mathrm{mL}$, and serum PTH: $104 \mathrm{pg} / \mathrm{mL}$ ). Hemogram was suggestive of hypochromic anemia and thrombocytopenia and peripheral smear revealed anisopoikilocytosis, tear drop, and fragmented red blood cells with anemia and thrombocytopenia; reticulocyte index was $1.2 \%$. An abdominal sonography revealed moderate hepatosplenomegaly. A bone marrow biopsy was advised; however, the family refused consent. Oral cholecalciferol therapy at 3,000 IU/day was initiated along with oral calcium of $500 \mathrm{mg} /$ day. Iron syrup was advised at received

August 27, 2020

accepted after revision

December 1, 2020
DOI https://doi.org/

$10.1055 / \mathrm{s}-0040-1722273$.

ISSN 2474-5871.
$6 \mathrm{mg} / \mathrm{kg} /$ day. On follow-up, the child showed remarkable clinical improvement in the form of gaining motor milestones and a significant reduction in organomegaly. At 2 months, the liver was no longer palpable and the spleen had reduced to 3-cm clinically. This was concurrent with a biochemical improvement in hemoglobin, platelets, phosphorus, and alkaline phosphatase levels.

The differential diagnosis in an infant with splenohepatomegaly, rickets, and bicytopenia included a hematological neoplasm, such as leukemia, a storage disorder, such as Gaucher's disease, as well as hyperparathyroidism-induced myelofibrosis. In an otherwise healthy baby without lymphadenopathy or atypical lymphocytes on peripheral smear and normal serum lactate dehydrogenase levels, a diagnosis of hematological neoplasm was unlikely. Likewise, absence of consanguinity, cherry red spot on fundus examination, as well as typical radiological features, such as Erlenmeyer's flask deformity, went against the diagnosis of a storage disorder. Reduced blood cell counts along with leucoerythroblastosis on peripheral smear are corroborative for the diagnosis of myelofibrosis and requires a histological confirmation. Characteristically, a bone marrow aspiration results in a dry tap with biopsy demonstrating hypocellular bone marrow with increased reticulin deposition. Although, in our child, a bone marrow biopsy could not be performed, the presence of vitamin-D deficiency, characteristic peripheral blood picture, normal reticulocyte index, and the rapid improvement of bicytopenia and organomegaly on oral cholecalciferol therapy favored the diagnosis of vitamin-D deficiency-associated myelofibrosis.

In the pediatric population, myelofibrosis commonly occurs secondary to neoplasms, such as leukemia and lymphoma, or to nonneoplastic conditions, such as vitamin-D deficiency, systemic lupus erythematosus, Fanconi's anemia, and hyperparathyroidism; primary myelofibrosis is rare. ${ }^{2}$

(c) 2021. The Author(s).

This is an open access article published by Thieme under the terms of the Creative Commons Attribution License, permitting unrestricted use, distribution, and reproduction so long as the original work is properly cited. (https://creativecommons.org/licenses/by/4.0/)

Georg Thieme Verlag KG, Rüdigerstraße 14, 70469 Stuttgart, Germany 
Children with myelofibrosis associated with vitamin-D deficiency typically present in infancy. Hyperparathyroidism resulting from vitamin-D deficiency is postulated to be responsible for the myelofibrosis. ${ }^{3}$ Increased parathormone levels lead to decreased erythropoietin receptors on erythroid progenitor cells and increased medullary fibrosis. The hepatosplenomegaly results from subsequent extramedullary hematopoiesis. Another possible pathophysiologic feature of vitamin-D deficiency induced myelofibrosis could involve reduced levels of active vitamin-D metabolites which are responsible for the maturation of the monocyte and megakaryocyte precursors leading to reduced degradation and increased deposition of collagen. ${ }^{4}$ Along with cellular differentiation, vitamin D also plays a role in cellular proliferation; children with vitamin $\mathrm{D}$-induced myelofibrosis have lower concentrations of CD34+ cells in bone marrow but increased levels in peripheral blood and splenic aspirates both of which return to normal with treatment of rickets. ${ }^{5}$

Previous published case reports mainly showed a partial or complete improvement of clinical, radiological, and hematologic parameters with vitamin-D therapy, irrespective of the regimen used. ${ }^{6}$ However, persistent pancytopenia and death due to septic shock have also been reported. ${ }^{2}$

Vitamin-D supplementation up to 1 year of age is routinely recommended. Also, secondary myelofibrosis must be kept in mind for any child presenting with rickets and hepatosplenomegaly. A high index of suspicion can obviate the need for extensive workup and support a more conservative approach toward management.

Conflict of Interest

None declared.

\section{References}

1 Sharma S, Jain R, Dabla PK. The role of 25-hydroxy vitamin D deficiency in iron deficient children of North India. Indian J Clin Biochem 2015;30(03):313-317

2 Balasubramanian S, Varadharajan R, Ganesh R, Shivbalan S. Myelofibrosis and vitamin $\mathrm{D}$ deficient rickets-a rare association. Indian Pediatr 2005;42(05):482-484

3 Elidrissy ATH. Myelofibrosis associated, with Rickets, is it hyperparathyroidism the triggering agent or vitamin $\mathrm{D}$ and hypocalcemia or hypophosphatemia. Int J Clin Endocrinol Metab 2016;2 (01):019-023

4 Atiq M, Fadoo Z, Naz F, Khurshid M. Myelofibrosis in severe vitamin D deficiency rickets. J Pak Med Assoc 1999;49(07): 174-177

5 Yetgin S, Yalçin SS. The effect of vitamin D3 on CD34 progenitor cells in vitamin D deficiency rickets. Turk J Pediatr 2004;46(02): 164-166

6 Bhakhri BK, Debata PK. Nutritional rickets presenting with myelofibrosis. Indian J Pediatr 2010;77(12):1437-1439 